Pub. Mat, UAB

no 10 , De sembre 1978

\title{
A NOTE ON THE ITERATION OF EXPONENTIAIS
}

Carles sins

Seccio de Matematiques

Universitat Autonoma de Barcelona

We consider the sequence $y_{i \phi 1}=z^{Y_{i}}, \quad i \geq 0, z \in R_{+}$, with

$y_{0}=z$. In [1], the question of the hehavior of such sequence is posed. Subsequently, many references to solutions are given (see [2]), for instance [3]. In this paper we obtain a full description of these iterates as functions of the parameter $z$, for eve ry value of $y$. Our technique just uses the discretedynamical sys tem in $R_{+}$defined by $f_{z}(x)=z^{x}$. The properties of the curves of fixed points and of two-periodic points are also given.

\section{\$1.- Fixed points.}

a) If $z>1, f_{z}(x)$ is concave; $z^{x}=x$ has solution iff $z \leq b$, where $b$ must satisfy $x=b^{x}, 1=b^{x} \ln b \Rightarrow b=e^{1 / e}$. If $z \varepsilon(1, b)$ thexe are two fixed points $x_{1}(z)<x_{2}(z)$. They coinci de for $z=b$.

For $x_{1}(z)$ we have $0<f^{\prime}\left(x_{1}(z)\right)<1$. Then it is stable. Ins tability occurs for $x_{2}(z)$.

b) If $z<1, f_{z}(x)$ is monotonically decreasing. Then $x=z^{x}$ has only one solution $x_{1}(z)$.

Stability: $f_{z}^{\prime}(x)<0$ implies $x_{1}(z)$ stable if $f_{z}^{\prime}\left(x_{1}\right)>-1$. The limit of stability is found at $x \ln z=-1 \Rightarrow x^{\prime} x=1 / e$, and $z_{\text {lim }}=a=e^{-\dot{e} \text {. }}$ 
For $z=1-\varepsilon, \varepsilon$ small enough: $z^{x_{1}}>1-\varepsilon \Rightarrow f_{z}^{\prime}\left(x_{1}\right)=0(\varepsilon) \ldots$ Then the fixed points is stable for $z \in[a, 1)$. The negative character of $f^{\prime}$ implies that the iterates alternate around the $f \underline{i}$ xed point.

c) Curve of fixed points: Consider the curve $x=x(z)$, $z e(0, b)$ given by $x=z^{x}$ (two branches if $z>1$ ); $x^{\prime}=\frac{d x}{d z}=$ $=\frac{x}{z(1-\ln x)}=\frac{\ln x}{z \ln z(1-\ln x)}$. One has $x^{\prime}=\infty$ at $z=b$. The upper branch has $x_{2}^{\prime}<0, z \in(1, b)$, and lower one gives $x_{1}^{\prime}(z)>0$ in $(0, b)$. We get as limiting values: $\lim _{\bar{z}^{\rightarrow} x^{+}} x_{i}^{\prime}=-\lim _{z \rightarrow 0^{+}} \frac{1}{z \ln z}=\infty$; $\lim _{z \rightarrow 1^{+}} x_{2}^{\prime}=-\infty ; \lim _{z \rightarrow 1} x_{1}^{\prime}=1$. We obtain for the second derivative $x^{\prime \prime}=\frac{x \ln x+x /(1-\ln x)}{z^{2}(1-\ln x)^{2}}$ zero values iff $\ln x=(1 \pm \sqrt{5}) / 2$. Then there are only two turning points: one, $x_{2}^{i}$, in $x_{2}(z)$ and the other, $x_{1}^{i}$, in $x_{1}(z)$ for some $z<1$. With this information we can plot $x(z)$. This is done in fig.1.

\section{\$2,- Periodic points.}

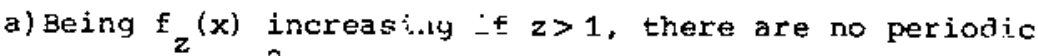
points. For $z<1, f_{z}^{2}(x)$ s also increasing. Then there are only fixed points under $f_{z}$ (studied in $\$ 1$ )) or 2-periodic points $x_{3}(z), x_{4}(z)$.

b) We consider the function $g_{z}(x)=z^{x}-\log _{2} x$ for $z<a$. we have $g_{z}\left(x_{1}\right)=0, g_{z}^{\prime}\left(x_{1}\right)<0$ and $g_{z}(1)>0$. Then there are points $y e\left(x_{1}, 1\right)$ fixed under $g_{z}$. Let us now show their uniqueness.

It is enough to proof that there is a unique point $x$ such that $g_{z}^{\prime}(x)=0$. Then $x z^{x} \ln ^{2} z=1$. We define $\Psi(x)=x z^{x}$. As $y^{\prime}(x)=(1+\ln x) z^{x}$, we have for $x>x_{1}:$ 


$$
|x \ln z|>\left|x_{1} \ln z\right|=\left|\ln x_{1}\right|>1 \Rightarrow \psi^{\cdot}(x)<0 \text { for } x \in\left(x_{1}, 1\right) .
$$
But $f_{z}^{\prime}\left(x_{1}\right)=z^{x_{1}} \ln z, \quad\left(f_{z}^{\prime}\left(x_{1}\right)\right)^{2}=x_{1} z^{x_{1}} \ln ^{2} z \Rightarrow \psi^{\prime}\left(x_{1}\right)=$ $=\left(f_{z}^{\prime}\left(x_{1}\right)\right)^{2} / 1 n^{2} z$. Then $g_{z}^{\prime}$ has a zero in $\left(x_{1}, 1\right)$ iff $x_{1}$ is unstable for $f_{z}$, i.e., iff $z \varepsilon(0, a)$. So there is only one 2-periodic point in $\left(x_{1}, 1\right)$ which is $x_{4}(z)$. The image under $f_{z}$ ' $x_{3}(z)$. is also 2-periodic and belongs to $\left(0, x_{1}\right)$. The stability of 2-periodic points is guaranteed because $g_{z}^{\prime}\left(x_{i}\right)>0, i=3,4$. Furthermore, if $h_{z}(x)=z^{z^{x}}$ we have $h_{z}^{r}\left(x_{i}\right)>0, i=3,4$.

c) Curve of two-periodic points: There are two branches for $z \in(0, a]$ which coincide if $z=a$. From $z^{x}=\frac{\ln x}{\ln z}$ we derive $z \ln z(x \ln x \ln z-1) x^{\prime}=-x \ln x(1+x \ln z)$. For $x \ln x \ln z=1$ we get $x^{\prime}=\infty$. This happens if $x=e^{-1}, z=a$. The signs of the factors allow us to state that $x_{3}^{\prime}>0, x_{4}^{\prime}<0$. Indeed, we begin by proving that $1+x \ln z$ has only one zero: $z^{x}=e^{-1}=\ln x / \ln z$ and $x \ln z=-1$ imply $x \ln x=-e^{-1}$, i.e., $x=e^{-1}$.

The same happens for $x \ln x \ln z-1$, but the proof is more tedious: $z^{x}=e^{1 / \ln x}=\ln x / \ln z$ and $x \ln x \ln z=1$ give us $x \ln ^{2} x=e^{1 / \ln x}$. The change $t=1 / \ln x$ transforms the above given condition to $\xi(t)=\xi\left(t^{-1}\right)$, where $\xi(t)=t e^{t}, t<0$. We must veri fy that $t=-1$ ia the unique solution. This is equivalent to find the positive solutions of $\phi(t)=t$, where $\phi(t)=\exp \left(\frac{1}{2}(t-1 / t)\right)$. Obviously 0,1 are solutions. But $\varphi^{\prime}(t)=\left(t^{2}+1\right) \oplus(t) /\left(2 t^{2}\right) ; \varphi^{\prime \prime}(t)=$ $=\left(t^{4}+2 t^{2}-4 t+1\right) \varphi(t) /\left(4 t^{4}\right) ; \phi^{\prime \prime \prime}(t)=\left(t^{6}+3 t^{4}-12 t^{3}+27 t^{2}-12 t+1\right) \varphi(t) / 8 t^{6} 9$. Then, $\varphi^{\prime}(0)<1, \varphi^{\prime}(1)=1, \varphi^{\prime \prime}(1)=0, \varphi^{\prime \prime}(1)>0$ implies that the numberof zeros of $\Phi(t)=t$ in $(0,1)$ counted with their multiplici ties is even. If that number is positive, $\Phi "(t)$ must have at least two zeros in $(0,1)$, but such zeros satisfy $t^{4}+2 t+1=4 t$. Since $\left(t^{2}+1\right)^{2}$ is concave, there are exactly 2 solutions and one of them is 1 . Then there are no solutions of $\varphi(t)=t$ in $(0,1)$. On the 
other side $\varphi^{\prime \prime \prime}>0$ if $t>1$, implies $\varphi(t)>t, \forall t>1$. This ends the proof.

The behavior of the two branches near $z=0$ is found by asymptotic expansions: Let $x_{3}=z(1+\alpha(z)), \alpha(z)=o(1)$. We try to satisfy $z^{x}=\ln x / \ln z$. Then $a(z)=z \ln n^{2} z+0\left(z^{2} \ln z\right)$. The ina ge under $f_{z}$ gives $x_{4}=1+z \ln z+0\left(z^{2} \ln ^{2} z\right)$. This allows us to plot $x_{i}(z), i=3,4$. See Eig.1.

\section{$\S 3 .-$ Behavior of the iterates.}

Let be $y_{0}, Y_{1}, Y_{2}, y_{3}, \ldots$ the successive iterates.

a) If $z>b$ one has $y_{n} \uparrow 00$.

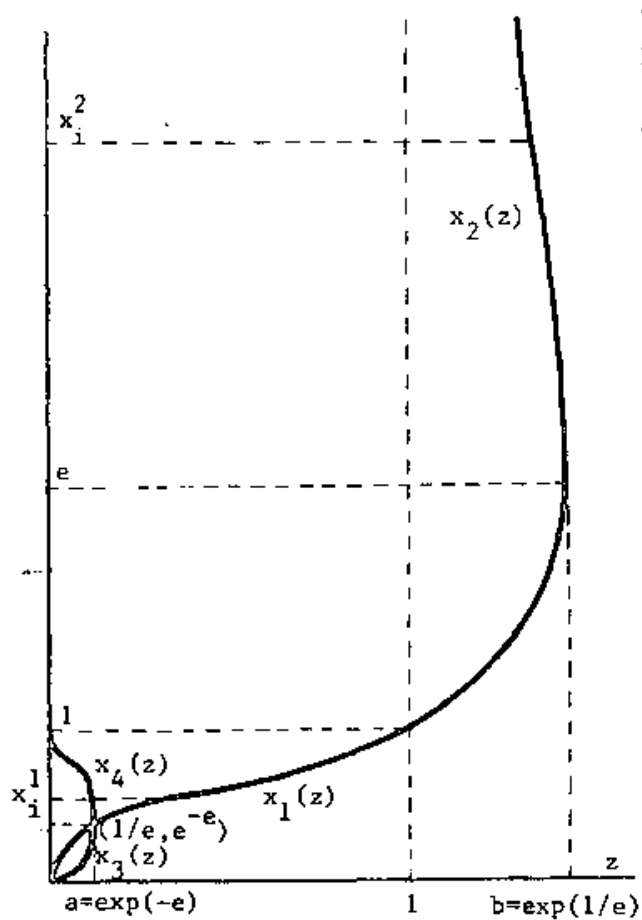

Fig.1 b) For $z=b$ and $y_{0} \leq e$, we have $y_{n} \hat{e}$. For $y_{0}>e \Rightarrow y_{n} \uparrow \infty$.

c) If $z \in(1, b)$ and $y_{0} \leq x_{1}(z)$ we get $y_{n} \uparrow x_{1}(z) ; y_{0} \varepsilon\left(x_{1}, x_{2}\right) \Rightarrow y_{n} \downarrow x_{1} ; y_{0}>x_{2}$ $\Rightarrow y_{n} \downarrow \infty$.

d) When $z \in[a, 1)$, for every initial. value $y_{0}$ we have $y_{n} \rightarrow x_{1}(z)$. but the iterates alternate in $\left(0, x_{1}\right),\left(x_{1}, \infty\right)$. so, $y_{0} \in\left(0, x_{1}\right) \Rightarrow y_{2 k} \nmid x_{1}, y_{2 k+1}+x_{1}$. For the critical value $z=a$ we have a bifurcation: $x_{1}(z)$ losses the stability and a two-point stable cycle appears.

e) If $z \notin(0, a)$ we have also the fixed point $x_{1}(z)$, but any $y_{0} \neq x_{1}(z)$ gives iterates converging to the cycle $3,4^{(2)}$ (and them they do not properly converge). 


$$
\left.y_{0} e\left(x_{3}, x_{1}\right) \Rightarrow y_{2 k+1}\right|_{4}, y_{2 k}\left|x_{3} ; y_{0} \varepsilon\left(0, x_{3}\right) \Rightarrow y_{2 k}+1\right| x_{4}, y_{2 k} \mid x_{3} .
$$

Similar results are obtained for $\mathrm{y}_{0} \varepsilon\left(\mathrm{x}_{1}, \mathrm{x}_{4}\right)$ or $\mathrm{y}_{0} \in\left(\mathrm{x}_{4}, \infty\right)$.

In particular, if $y_{0}=z$ the iterates converge to $x_{1}$ iff $z \varepsilon[a, b]$ and to the cycle $\left\{x_{3} x_{4}\right\}$ iff $z<a$.

\section{References}

[1] Notices Amer. Math. Soc. 25(1978), 197.

[2] Notices Amer. Math. Soc. 25(1978), 253, 335.

[3] Bromwich, T.J.I'A.: "An Introduction to the Theory of Infinite Series", MacMillan, 1965, p. 23. 